\title{
Modified Encircling Scleral Buckle Technique Without Subretinal Fluid Drainage or Retinopexy
}

\author{
Mostafa Mafi · Masoud Mirghorbani · Hossein Ghahvehchian • \\ S. Saeed Mohammadi · Hamid Riazi-Esfahani - Elias Khalili Pour • \\ Alireza Mahmoudi · Hassan Khojasteh · Bobeck S. Modjtahedi
}

Received: May 19, 2020 / Published online: July 14, 2020

(C) The Author(s) 2020

\section{ABSTRACT}

Introduction: Scleral buckling (SB) tends to be more challenging and time-consuming for compared to the pars plana vitrectomy for repairing rhegmatogenous retinal detachments (RRDs). This study characterizes a novel and simplified technique for SB.

Digital Features To view digital features for this article go to https://doi.org/10.6084/m9.figshare.12547403.

Electronic supplementary material The online version of this article (https://doi.org/10.1007/s40123$020-00279-1$ ) contains supplementary material, which is available to authorized users.

M. Mafi · M. Mirghorbani · H. Ghahvehchian .

S. S. Mohammadi · H. Riazi-Esfahani .

E. Khalili Pour · A. Mahmoudi · H. Khojasteh ( $₫)$

Department of Ophthalmology, Farabi Eye Research

Center, Farabi Eye Hospital, Tehran University of

Medical Sciences, Tehran, Iran

e-mail: khojaste_hasan@yahoo.com

\section{B. S. Modjtahedi}

Department of Ophthalmology, Southern California

Permanente Medical Group, Baldwin Park, CA, USA

B. S. Modjtahedi

Department of Research and Evaluation, Southern

California Permanente Medical Group, Pasadena,

CA, USA

B. S. Modjtahedi

Eye Monitoring Center, Kaiser Permanente

Southern California, Baldwin Park, CA, USA

e-mail: bobmodj@gmail.com
Methods: In this single-masked randomized interventional study, patients with RRDs who were eligible for SB were randomly assigned to either the standard (S) or modified (M) technique of SB. In the modified approach, neither intraoperative break localization nor cryopexy or subretinal fluid drainage was done. A large tire $(276 / 279)$ was placed where preoperative retinal breaks had been localized with a 240 encircling band placed for support of the remaining retina. Patients were followed for 12 months and the primary outcomes were differences between the surgical groups in operative time, anatomical success, visual acuity, and complication rate.

Results: Thirty-six eyes were included in the study (18 in each arm). There were no differences in baseline patient demographics or characteristics including gender, age, lens and macular status, preoperative vision, and symptom duration. The mean length of surgery was $72.2 \pm 13.2$ and $56.2 \pm 9.5 \mathrm{~min}$ in groups $\mathrm{S}$ and $\mathrm{M}$, respectively $(P=0.001)$. Complete retinal reattachment at the end of month 12 after single surgery was $80.6 \%$ overall; $77.8 \%(14 / 18)$ in group $S$ and $83.3 \%(15 / 18)$ in group $M$ $(P>0.999)$. After 12 months, both groups achieved similar final best-corrected visual acuity (BCVA): $0.26 \pm 0.23$ and $0.23 \pm 0.17$ logMAR in groups $S$ and $M$, respectively $(P=0.231)$. Controlling for preoperative BCVA on ANCOVA testing, there were no significant differences in visual improvement between the 
two groups $[F(1,26)=0.02, P=0.966(95 \% \mathrm{CI})$ -0.128 to 0.123$)]$. Scleral perforation $(2: 1)$, vitreous hemorrhage (3:2), and transient rise of intraocular pressure (3:4) all occurred at a low and similar rate between the two groups (S:M). Conclusion: Modified SB technique was noninferior compared to the standard approach for anatomical and visual outcomes. Shortening surgical time while maintaining low complication rates makes this an appropriate approach to SB, especially for vitreoretinal surgery trainees.

Keywords: Cryopexy; Retinal detachment; Retinopexy; Scleral buckle; Surgery; Technique

\section{Key Summary Points}

\section{Why carry out this study?}

There is a downward trend in popularity of scleral buckling (SB) due to its more challenging and time-consuming nature compared to pars plana vitrectomy.

We aimed to provide a simplified and more user-friendly technique which may help surgeons become more comfortable with SB.

\section{What was learned from the study?}

SB can be modified with the elimination of cryotherapy and subretinal fluid drainage, as well as less dependence on intraoperative indirect ophthalmoscopy.

The proposed modified technique is noninferior to the more standard SB technique for both visual and anatomical outcomes.

Less high-risk maneuvers in this modified technique may reduce the risk of complications, especially in more novice surgeons.

\section{INTRODUCTION}

The approach to repairing rhegmatogenous retinal detachments (RRDs) can vary depending on features of the detachment as well as the surgeon's comfort level [1-3]. Small gauge pars plana vitrectomy (PPV) has grown in popularity such that many surgeons view it as the first-line treatment for most detachments $[4,5]$. Scleral buckling (SB) has followed a commensurate downward trend in popularity because many surgeons perceive it as more technically challenging and time-consuming. Additionally, complications of SB including ocular ischemia, diplopia, and the risk of retinal incarceration have caused some surgeons to move away from this technique $[6,7]$. Many surgeons consider SB to be a dying art, with younger surgeons often not feeling comfortable with this technique and older surgeons often expressing the belief that their SB skills have diminished from a lack of practice. It is the "art" of buckling that many surgeons find frustrating; intraoperative endpoints such as buckle height, amount of cryotherapy to apply, and subretinal fluid drainage are often difficult to quantify. The surgical success rates for PPV and SB are comparable, and while SB is generally considered to be more labor-intensive for the surgeon, it requires less complicated surgical equipment and is associated with a lower cost when compared to PPV [8-11].

We sought to develop a more standardized approach for SB that would limit the subjective elements of this surgery while shortening surgical time and maintaining low complication rates in an effort to create a more user-friendly approach that can be employed by surgeons with varying degrees of experience. Herein we report the results of this new technique and compare it to a traditional method of SB.

\section{METHODS}

This was a prospective interventional case series conducted at Farabi Eye Hospital, Tehran University of Medical Sciences, a tertiary ophthalmology center in Iran. The study protocol was approved by the Institutional Review Board 
of the Farabi Eye Hospital and the study adhered to the Declaration of Helsinki. Eligible patients participated after providing written informed consent.

Patients with RRDs amenable to SB seen between May 1, 2017 to January 1, 2018 were included. A detailed history was taken from all patients who also received a complete ophthalmic examination preoperatively including best-corrected visual acuity (BCVA), intraocular pressure measurements, slit-lamp examination, and dilated fundus examinations. Snellen acuity taken in the clinic was converted into logarithm of the minimum angle of resolution (logMAR) for the purpose of statistical analysis. Special attention was placed on the status of the retina [macular status, presence of proliferative vitreoretinopathy (PVR), clock-hours detached, and number/location of breaks] and any possible media opacities including the lens status.

Retinal detachment in eyes with pathologies such as break or lattice degeneration in more than two quadrants which were not suitable to be managed by segmental sponge or pneumatic retinopexy, including those with either localized detachments or total retinal detachments, were included. Eyes were excluded from this study if there was poor visualization of the retina from any causes (e.g., cataract, vitreous hemorrhage, corneal opacity), giant retinal tears, posterior retinal breaks not amenable to buckling, no breaks visualized, previously failed primary SB or pneumatic retinopexy, grade $\mathrm{C}$ PVR or worse, or any prior history of ocular disease that would affect vision including amblyopia, macular degeneration, optic neuropathy, retinal vascular occlusion, or visionthreatening forms of diabetic retinopathy.

Patients were randomly assigned to one of two intervention arms using a numerical random generator. Group S received standard SB with cryopexy of retinal breaks, whereas group $M$ received a modified surgical approach. All the surgeries were performed by second-year vitreoretinal surgery fellows as a part of their fellowship training program under supervision of an expert surgeon (H.K.).

Primary outcome measures were operative time, anatomic success (single surgery successful retinal reattachment) at month 12 , and
BCVA at month 12. Secondary outcome was complication rate during the follow-up period. The time of the surgery recorded for each patient was defined as the time between the start of the conjunctival peritomy to the placement of the last conjunctival suture.

\section{Overview of Current Procedure (Group S)}

After the eye was prepped and draped in the standard ophthalmic manner, a $360^{\circ}$ conjunctival peritomy was performed, followed by isolation of the four rectus muscles with silk suture. The external globe was examined for scleral thinning and abnormal vortex veins. A complete $360^{\circ}$ scleral depressed examination was done with marking of the retinal breaks and cryopexy of the breaks. Then two mattress sutures with 5.0 MERSILENE ${ }^{\circledR}$ were pre-placed in each quadrant. In quadrants with a tire, the anterior suture pass was usually made at the level of muscle insertion with possible location adjustment to provide support to visualized breaks. The posterior suture pass was made $10 \mathrm{~mm}$ posterior to the anterior suture pass. In quadrants with only a band, the anterior and posterior suture passes were made approximately 12 and $16 \mathrm{~mm}$ posterior to the limbus, respectively. The placement of the buckle was done such that the break would ideally fall on the posterior $1 / 3$ of the buckle.

An encircling silicone tire $(276 / 279$, Mira Inc., Uxbridge, MA, USA) was placed in the quadrants of the causative break and peripheral pathologies such as lattice degeneration (usually not $360^{\circ}$ ) as well as under the corresponding rectus muscle and the pre-placed nonabsorbable sutures. A 240-silicone encircling band was placed through the groove of the tire and circumferentially $360^{\circ}$ (under the rectus muscles and pre-placed sutures). If needed subretinal fluid drainage was performed, preferably at a site beneath the buckle. The tire and band were secured to the sclera by tying the preplaced sutures. Then two ends of the band were passed through a loop of releasable 5.0 nonabsorbable suture. Indirect ophthalmoscopy was done to confirm appropriate support of the break with adjustment of the buckle until it 
achieved the desired height after which the loop of suture was tightened. The excess ends of the band were cut $2-3 \mathrm{~mm}$ away from the suture. The conjunctiva was closed with 5.0 polyglactin 910 (Vicryl $\left.^{\circledR}\right)$ suture.

\section{Modification of Procedure (Group M)}

After isolation of the muscles with silk suture and external inspection of the globe, a single mattress suture with 5.0 non-absorbable suture was placed in the center of each quadrant without localization of the causative breaks, except for the final quadrant in which the two mattress sutures were placed to secure the ends of SB (Fig. 1). In quadrants that would have a tire, the anterior pass was made at the level of the muscle insertion and the posterior pass $10 \mathrm{~mm}$ away, while in quadrants with only a band the sutures were placed as previously described in group $S$. Then the silicone tire (276/279) and 240 band were passed under the rectus muscles and pre-placed sutures which were tightened to secure the buckle. The tire was placed in quadrants with corresponding retinal detachments and peripheral pathologies that were seen in clinic while the 240 band was circumferentially $360^{\circ}$. Similar to the standard technique, the two free ends of the buckle met in the final quadrant and were passed through a

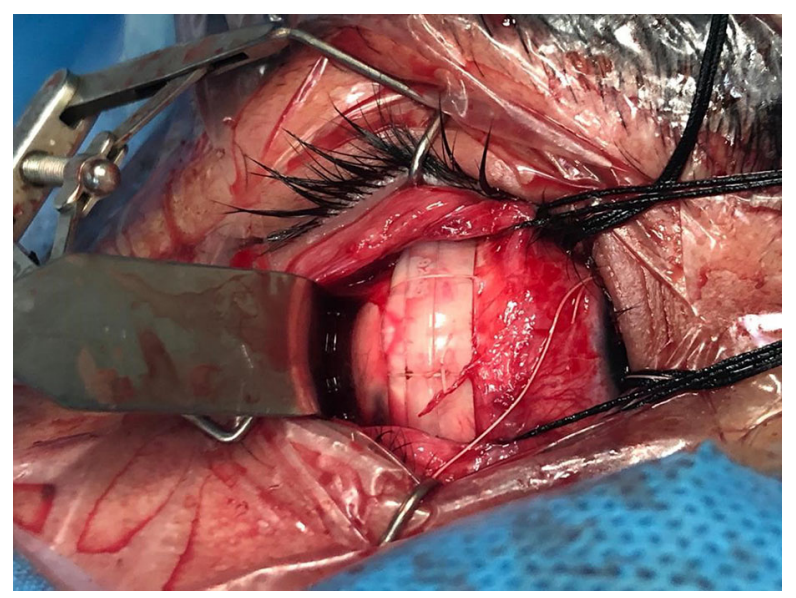

Fig. 1 After isolation of the muscles and placement of silk suture, the encircling tire and band was secured with one mattress suture with non-absorbable suture at the center of each quadrant (inferotemporal quadrant is shown) loop of releasable non-absorbable suture. Then, $0.1 \mathrm{ml}$ of aqueous humor was removed and the buckle was tightened up to the point where the intraocular pressure normalized on tactile inspection at which point the loop of releasable suture was tightened. Excess buckle length was then cut $2-3 \mathrm{~mm}$ away from the non-absorbable suture. No cryopexy or subretinal fluid drainage was performed during the procedure. The retina was visualized only at the end of the surgery with indirect ophthalmoscopy to ensure patency of the central retinal artery.

The schematics of standard and modified SB approaches are shown in Fig. $2 \mathrm{a}$ and b, respectively.

\section{After Surgery}

Patients were treated with chloramphenicol eyedrops every $6 \mathrm{~h}$ for 1 week and betamethasone $1.0 \%$ every $4 \mathrm{~h}$ for 1 week, after which the dose was tapered during 3 weeks. Patients were followed postoperatively for at least 12 months. Data collectors were masked as to each patient's surgical arm. During the follow-up period, any new or previously undiagnosed posterior breaks were treated with barrier laser.

\section{Data Analysis}

Mean, standard deviation, range, frequency, and percentage were used to describe data. Chisquare, Fisher exact, Mann-Whitney, and $t$ tests were used to compare differences between groups. Analysis of covariance (ANCOVA) was used to determine any significant differences in adjusted means. To assess the predictive effect of possible dependent variables on postoperative BCVA, a non-parametric regression model was tested. Statistical analysis was performed using SPSS software (IBM Corp. Released 2017. IBM SPSS Statistics for Windows, Version 25.0. Armonk, NY: IBM Corp.). $P$ values of 0.05 or less were considered statistically significant. 


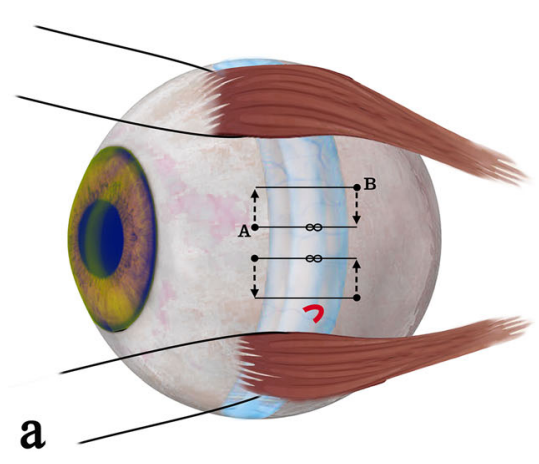

b

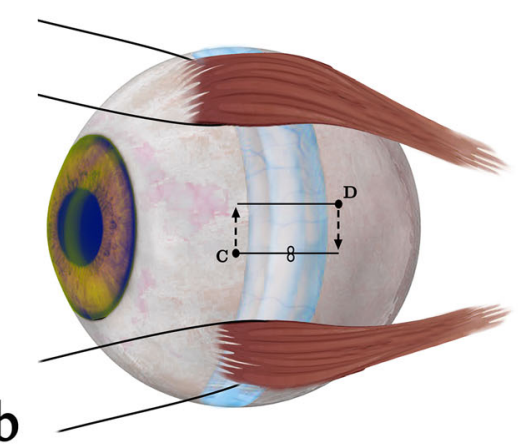

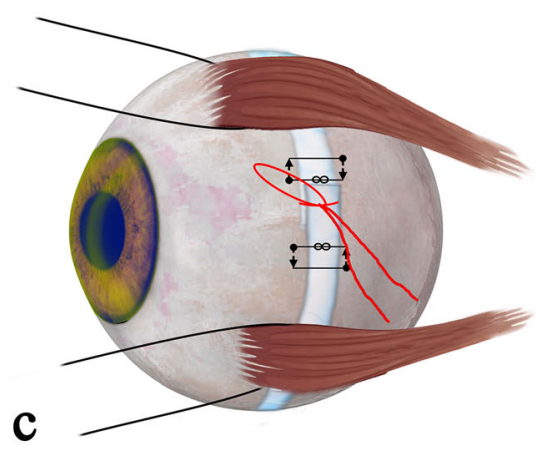

Fig. 2 Schematic representation of standard and modified scleral buckling. In routine procedure (a), the break is localized by indirect ophthalmoscopy intraoperatively. Then two mattress sutures are placed accordingly to ensure that the underlying tire covers the break entirely, such that the break would fall on the posterior $1 / 3$ of the buckle. In the modified technique (b), no localization of the break is done, and only one mattress suture is placed in the quadrant. At the final quadrant, in both techniques, the two mattress sutures were placed to secure the ends of SB to the sclera (c); for the placement of the 240 band, the anterior and posterior suture passes were made approximately 12 and $16 \mathrm{~mm}$ posterior to the limbus, respectively. After passing SB under the rectus muscles and the preplaced sutures, the two ends of the band were passed through a loop of releasable 5.0 non-absorbable suture. Appropriate tightness of the SB was determined either by indirect ophthalmoscopy in the standard technique or normalization of intraocular pressure after removing $0.1 \mathrm{ml}$ of aqueous humor in the modified technique

surgery anatomic success, defined as complete retinal reattachment, at the end of month 12 was $80.6 \%(29 / 36)$ for all patients: $77.8 \%(14 /$ $18)$ in group $S$ and $83.3 \%(15 / 18)$ in group $M$ $(P>0.999)$. No significant differences in reattachment rates were observed between the two groups at the end of our follow-up. Postoperatively anatomic success was achieved in all macula-on RRDs (9/9) and in 74.1\% (20/27) of macula-off RRDs $(P=0.156)$. Patients who did not achieve anatomic success ( $n=7$, all maculaoff RRDs) had a longer mean duration of symptoms ( $24.4 \pm 8.8$ days) compared to those who achieved anatomic success $(9.7 \pm 7.3$ days, $P<0.001$ ), with no other variables being significantly different between the detached retina and attached retina groups (Table 2).

Postoperative logMAR BCVA at month 12 was not significantly different between surgical intervention groups when controlling for preoperative logMAR BCVA on ANCOVA testing $[F(1,26)=0.02, P=0.966(95 \% \mathrm{CI})-0.128$ to $0.123)]$. The prediction effect of preoperative vision, age, sex, lens status, duration of symptoms before surgery and surgical method on postoperative vision at 12 months were analyzed and only preoperative vision was a 
Table 1 Baseline characteristics

\begin{tabular}{|c|c|c|c|}
\hline & Group M & Group S & $P$ value \\
\hline Number & 18 & 18 & \\
\hline Male: Female & $11: 7$ & $9: 9$ & $0.505^{\mathrm{a}}$ \\
\hline Mean age & $45.7 \pm 10.4$ years $(28-63$ years $)$ & $44.5 \pm 11.6$ years $(22-63$ years $)$ & $0.754^{\mathrm{b}}$ \\
\hline Phakic: Pseudophakia & $15: 3$ & $14: 4$ & $>0.999^{c}$ \\
\hline Macula on: Macula off & $14: 4$ & $13: 5$ & $>0.999^{\mathrm{c}}$ \\
\hline Symptom duration & $13.1 \pm 10.7$ days & $12.0 \pm 9.0$ days & $0.752^{b}$ \\
\hline $\begin{array}{l}\text { Mean pre-op baseline visual } \\
\text { acuity }\end{array}$ & $\begin{array}{l}\log \text { MAR } 1.14 \pm 0.64(\text { Snellen } \\
\text { equivalent, } 6 / 83)\end{array}$ & $\begin{array}{l}\log \text { MAR } 1.01 \pm 0.61 \text { (Snellen } \\
\quad \text { equivalent, } 6 / 62)\end{array}$ & $0.639^{\mathrm{b}}$ \\
\hline
\end{tabular}

a Chi-square test

b Independent $t$-test

c Fisher's exact test

Table 2 Characteristics based on retinal status at final follow-up

\begin{tabular}{llll}
\hline & Attached & Unattached & $P$ value \\
\hline Number & 29 & 7 & $>0.999^{\mathrm{a}}$ \\
Male: Female & $17: 12$ & $3: 4$ & $0.611^{\mathrm{b}}$ \\
Mean age & $45.55 \pm 11.16$ years & $43.43 \pm 10.60$ years & $>0.999^{\mathrm{a}}$ \\
Phakic: Pseudophakia & $23: 6$ & $6: 1$ & $>0.999^{\mathrm{a}}$ \\
Group M: Group S & $15: 14$ & $3: 4$ & $0.156^{\mathrm{a}}$ \\
Macula off: Macula on & $20: 9$ & $7: 0$ & $<0.001^{\mathrm{b}}$ \\
Symptom duration & $9.72 \pm 7.34$ days & $24.43 \pm 8.83$ days & $0.102^{\mathrm{b}}$ \\
Mean preoperative BCVA & $\log$ AAR $0.99 \pm 0.63$ & $\log$ AR $1.44 \pm 0.45$ & \\
\hline
\end{tabular}

a Fisher's exact test

b Mann-Whitney test

significant predictor $(\beta=0.195, \quad P=0.005$, Table 3$)$. There was a significant improvement in visual acuity at the final follow-up for all the cases where anatomical reattachment was achieved from $1.08 \pm 0.62$ (Snellen equivalent, $6 / 72$ ) preoperatively to $0.25 \pm 0.20 \log$ MAR (Snellen equivalent, 6/11) $(P<0.001)$. Both surgical groups achieved similar final visual acuity: $0.26 \pm 0.23$ logMAR (Snellen equivalent, 6/11) and $0.23 \pm 0.17 \log$ MAR (Snellen equivalent, 6/10) in groups $S$ and $M$ respectively $(P=0.231)$.
Complication rates were comparable between groups: scleral perforation from suture placement occurred in one group $\mathrm{M}$ eye and two group $\mathrm{S}$ eyes. Vitreous hemorrhage $(\mathrm{VH})$ occurred in two group $\mathrm{M}$ eyes and three group $\mathrm{S}$ eyes, with PPV being necessary in one eye of group $S$ as a result of the development of highgrade PVR with retinal detachment after VH had cleared. All other VHs cleared without the need for intervention. Four eyes $(22.2 \%)$ in group $\mathrm{M}$ and $3(16.7 \%)$ eyes in group S developed transient rise of intraocular pressure, all of 
Table 3 Predictive effect of variables for postoperative logMAR best corrected visual acuity by a non-parametric regression model

\begin{tabular}{llll}
\hline Variable & $\boldsymbol{\beta}$ & $\boldsymbol{P}$ value & 95\% confidence interval \\
\hline Preoperative logMAR BCVA & 0.195 & 0.005 & 0.096 to 0.295 \\
Symptom duration & 0.002 & 0.699 & -0.010 to 0.014 \\
Age & 0.002 & 0.546 & -0.008 to 0.004 \\
Lens status & 0.057 & 0.682 & -0.342 to 0.228 \\
Gender & -0.040 & 0.807 & -0.375 to 0.296 \\
Surgery method & 0.228 & 0.176 & -0.112 to 0.569 \\
\hline
\end{tabular}

which were managed medically by intraocular pressure-lowering agents.

SB failure occurred in seven cases: three (two in group $\mathrm{M}$ and one in group $\mathrm{S}$ ) due to new or previously undiagnosed posterior breaks, two (one in group $\mathrm{M}$ and one in group $\mathrm{S}$ ) due to the development of high-grade PVR posterior to the buckle, one (group S) following subretinal hemorrhage secondary to scleral perforation which was initially managed with gas injection and position with later need for PPV, and one (group S) due to choroidal hemorrhage that occurred during subretinal fluid drainage. There were no cases of recurrent retinal detachment after initially successful anatomic results. Seven SB failures never achieved reattachment by SB and subsequently underwent PPV. There were no instances of buckle migration or extrusion during the follow-up period.

\section{DISCUSSION}

PPV has gained significant popularity for the treatment of RRD since its inception $[12,13]$. Several reasons have been postulated to be behind the corresponding decline in SB including the higher rate of ocular surface redness and inflammation compared to PPV, more ambiguous intraoperative endpoints, and concerns about SB risks such as intractable diplopia. Furthermore, retinal visualization can be challenging and time-consuming during SB when compared to small gauge PPV. Since PPV is an appropriate first-line option for many detachments, clinicians often find their comfort with SB diminishes over time as they fall out of practice or they feel their exposure during fellowship was inadequate. Nonetheless, SB still has a role in RRD repair and is often the preferred approach, especially in young phakic myopes where visual outcomes and the lack of cataract formation favor SB over PPV [9, 14]. Further, the lack of need for complex surgical equipment and the advantageous cost profile also favor the use of SB in many practice settings $[15,16]$.

SB has undergone innumerable adaptions since its introduction in the 1950s with variations in surgical technique, materials, and instruments. Reducing operative times and surgical complexity may help novice surgeons adopt SB. Attempts to innovate this surgery continue in an effort to expand its adoption by surgeons [17], including efforts to simplify retinal visualization with the use of fiber-optic endo-illumination, transscleral fiber-optic, or twin un-cannulated 27-gauge chandelier light source $[18,19]$. However, not only are these devices not available everywhere but they also require making an intraocular incision.

Unlike the $2 \pi \mathrm{R}$ (or "Vavvas") buckle, the technique described herein does not use a tire circumferentially around the eye, nor does it utilize cryotherapy (with indirect ophthalmoscopy). Additionally, buckle tightening is based on tactile intraocular pressure as 
described above rather than using a uniform amount of buckle trimming [20].

The modified SB outlined herein sought to reduce the number of surgical steps to lessen operative times and surgical complexity while maintaining visual and anatomic outcomes. Key features of this approach include the elimination of cryopexy which can be time-consuming and relies on indirect ophthalmoscopy for localization of the breaks and the elimination of subretinal fluid drainage which mitigates the risk of retinal perforation and incarceration, especially for inexperienced surgeons. This modified SB demonstrated comparable anatomic and visual outcomes with significantly shorter operative times when compared to the standard SB approach. The success rates in groups $\mathrm{S}$ and $\mathrm{M}$ were $77.8 \%$ and $83.3 \%$, respectively, which are comparable with the previously documented success rates of $74-94 \%$ quoted in the literature $[9,21,22]$.

Almost all intraoperative complications in scleral buckling such as scleral perforation, retinal incarceration, and vitreous hemorrhage are due to suturing process or subretinal drainage, so limiting these steps can help reduce the risk of complications. The mattress sutures do not contribute to achieving a buckling effect; instead, this is accomplished from tightening the buckle. The mattress sutures serve to hold the buckle in place and prevent migration, which is why only one suture is necessary per quadrant. Many surgeons find subretinal fluid drainage to be the most unnerving step in SB, given that the complications of this step can be significant and difficult to correct [23]. La Heij et al. reported a 3.8\% risk of hemorrhagic complications from subretinal fluid drainage at the time of SB [24]. We believe that this timeconsuming and potentially risky maneuver can be safely omitted from the SB procedure without affecting the final results in many patients. Although this study was not powered to detect differences in complications rates, the rate of complications in more novice surgeons may be lower with the modified SB technique since it has less high-risk maneuvers.

SB failures were most commonly from new or missed posterior breaks and PVR in this series. The rate of PVR $(5.56 \%)$ is comparable to previously published estimates of $5-10 \%$ and $5-20 \%$ in phakic and pseudophakic eyes after $\mathrm{SB}$, respectively $[24,25]$. Cryotherapy is considered a risk factor for PVR [26, 27]; however, our study was underpowered to detect differences in the rate of PVR between surgical groups. Nonetheless, eliminating cryotherapy did not adversely affect surgical success rates in our series, and given the potential role of cryotherapy in PVR formation, there may be a benefit avoiding this step in some patients. In this technique, neither intraoperative laser nor break localization was done. Placement of the buckle was determined based on preoperative examination. However, during the follow-up period, patients with new or previously undiagnosed posterior breaks were treated with barrier laser. Although during the 12 months follow-up we did not detect any redetachment or significant buckling effect reduction in clinical exam, Schwartz et al. reported the potential reduction of the buckling effect with time [28]. We did not apply the laser postoperatively once the retina flattened although this could be considered as an additional modification based on surgeon preference in the future. However, it should be noted that in extensive retinal pathologies, application of laser may be associated with increased risk of epiretinal membrane formation similar to cryotherapy [29]. Hence, postoperative laser application should be used with caution.

The analysis presented herein found that preoperative BCVA was the only significant predictor of final BCVA, which underscores that the exact technique employed is less important than treating patients in a timely fashion before visual deterioration takes place.

There are several limitations to this study. The small sample size limits the statistical analysis as a result of underpowering, especially for rare events such as PVR and intraoperative complications. Longer follow-up of the patients could also help further the understanding of later complications such as buckle migration or extrusion. There are innumerable variations in RRD presentation including chronicity, number/location of breaks, extent of detachment, phakic status, and the presence of PVR, which makes comparative surgical series difficult to 
interpret since it is not possible to adjust for all possible confounders. There is no universal or "one size fits all" approach for RRD repair; surgeons need to weigh the various clinical features when determining the best approach for their patient. The presented modified SB technique provides surgeons with a simplified yet effective method for SB.

\section{CONCLUSION}

Simplifying SB as proposed, with elimination of cryotherapy and subretinal fluid drainage, is non-inferior to the standard method of SB while requiring less operative time. Complication rates were low in this series where surgeries were performed by a second-year vitreoretinal surgery fellow, with the ease of implementing this technique making it ideal for trainees or those with limited exposure to SB surgery.

\section{ACKNOWLEDGMENTS}

We thank the participants of the study. Graphics were provided by Mr. Ghazi, audiovisual technician of Farabi Eye Hospital.

Funding. No funding or sponsorship was received for this study or publication of this article.

Authorship. All named authors meet the International Committee of Medical Journal Editors (ICMJE) criteria for authorship for this article, take responsibility for the integrity of the work as a whole, and have given their approval for this version to be published.

Authorship Contributions. Concept and design: Hassan Khojasteh, Mostafa Mafi, Masoud Mirghorbani; data acquisition: Hossein Ghahvehchian, Saeed Mohammadi, Alireza Mahmoudi, Hamid Riazi-Esfahani, Elias Khalili Pour; data analysis/interpretation: Mostafa Mafi, Masoud Mirghorbani, Bobeck S. Modjtahedi; drafting manuscript: Hossein Ghahvehchian, Saeed Mohammadi, Alireza Mahmoudi, Hamid Riazi-Esfahani, Elias Khalili Pour; critical revision of manuscript: Mostafa Mafi, Masoud Mirghorbani, Bobeck S. Modjtahedi, Hassan Khojasteh; supervision: Hassan Khojasteh, Masoud Mirghorbani. All authors read and approved the final manuscript.

Disclosures. Mostafa Mafi, Masoud Mirghorbani, Hossein Ghahvehchian, Saeed Mohammadi, Hamid Riazi-Esfahani, Elias Khalili Pour, Alireza Mahmoudi, and Hassan Khojasteh had nothing to disclose. Bobeck S. Modjtahedi receives research support from Genentech. Bobeck S. Modjtahedi is also a member of the journal's Editorial Board.

Compliance with Ethics Guidelines. The study protocol was approved by the Institutional Review Board of the Farabi Eye Hospital and the study adhered to the Declaration of Helsinki. Eligible patients participated after providing written informed consent.

Data Availability. The datasets generated during and/or analyzed during the current study are available from the corresponding author on reasonable request.

Open Access. This article is licensed under a Creative Commons Attribution-NonCommercial 4.0 International License, which permits any non-commercial use, sharing, adaptation, distribution and reproduction in any medium or format, as long as you give appropriate credit to the original author(s) and the source, provide a link to the Creative Commons licence, and indicate if changes were made. The images or other third party material in this article are included in the article's Creative Commons licence, unless indicated otherwise in a credit line to the material. If material is not included in the article's Creative Commons licence and your intended use is not permitted by statutory regulation or exceeds the permitted use, you will need to obtain permission directly from the copyright holder. To view a copy of this licence, visit http://creativecommons.org/licenses/bync/4.0/. 


\section{REFERENCES}

1. Heimann H, Hellmich M, Bornfeld N, Bartz-Schmidt KU, Hilgers RD, Foerster MH. Scleral buckling versus primary vitrectomy in rhegmatogenous retinal detachment (SPR Study): design issues and implications. SPR Study report no. 1. Graefes Arch Clin Exp Ophthalmol. 2001;239(8):567-74.

2. Sodhi A, Leung L-S, Do DV, Gower EW, Schein OD, Handa JT. Recent trends in the management of rhegmatogenous retinal detachment. Surv Ophthalmol. 2008;53(1):50-67.

3. Velez-Montoya R, Jacobo-Oceguera P, Flores-Preciado J, et al. Primary repair of moderate severity rhegmatogenous retinal detachment: a critical decision-making algorithm. Med Hypothesis Discov Innov Ophthalmol. 2016;5(1):18-311.

4. Hwang JC. Regional practice patterns for retinal detachment repair in the United States. Am J Ophthalmol. 2012;153(6):1125-8.

5. McLaughlin MD, Hwang JC. Trends in vitreoretinal procedures for medicare beneficiaries, 2000 to 2014 . Ophthalmology. 2017;124(5):667-73.

6. Reeves M-G, Pershing S, Afshar AR. Choice of primary rhegmatogenous retinal detachment repair method in US commercially insured and Medicare Advantage patients, 2003-2016. Am J Ophthalmol. 2018;196:82-90.

7. Shanmugam PM, Ramanjulu R, Mishra KCD, Sagar P. Novel techniques in scleral buckling. Indian $\mathrm{J}$ Ophthalmol. 2018;66(7):909-15.

8. Brazitikos PD, Androudi S, Christen WG, Stangos NT. Primary pars plana vitrectomy versus scleral buckle surgery for the treatment of pseudophakic retinal detachment: a randomized clinical trial. Retina. 2005;25(8):957-64.

9. Heimann H, Bartz-Schmidt KU, Bornfeld N, Weiss C, Hilgers RD, Foerster MH. Scleral buckling versus primary vitrectomy in rhegmatogenous retinal detachment: a prospective randomized multicenter clinical study. Ophthalmology. 2007;114(12): 2142-54.

10. Schaal S, Sherman MP, Barr CC, Kaplan HJ. Primary retinal detachment repair: comparison of 1-year outcomes of four surgical techniques. Retina. 2011;31(8):1500-4.

11. Schwartz SG, Flynn HW. Pars plana vitrectomy for primary rhegmatogenous retinal detachment. Clin Ophthalmol. 2008;2(1):57-63.
12. Gupta B, Neffendorf JE, Williamson TH. Trends and emerging patterns of practice in vitreoretinal surgery. Acta Ophthalmol. 2018;96(7):e889-e890.

13. Ramulu PY, Do DV, Corcoran KJ, Corcoran SL, Robin AL. Use of retinal procedures in medicare beneficiaries from 1997 to 2007. Arch Ophthalmol. 2010;128(10):1335-400.

14. Wong CW, Yeo IY, Loh BK, et al. Scleral buckling versus vitrectomy in the management of macula-off primary rhegmatogenous retinal detachment: a comparison of visual outcomes. Retina. 2015;35(12):2552-7.

15. Abdullah AS, Jan S, Qureshi MS, Khan MT, Khan MD. Complications of conventional scleral buckling occuring during and after treatment of rhegmatogenous retinal detachment. J Coll Physicians Surg Pak. 2010;20(5):321-6.

16. Ryan EH Jr, Mittra RA. Scleral buckling vs vitrectomy: the continued role for scleral buckling in the vitrectomy era. Arch Ophthalmol. 2010;128(9): 1202-5.

17. Kita M, Fujii Y, Kawagoe N, Hama S. Scleral buckling with a noncontact wide-angle viewing system in the management of retinal detachment with undetected retinal break: a case report. Clin Ophthalmol. 2013;7:587-9.

18. Tornambe PE, Hilton GF, Brinton DA, et al. Pneumatic retinopexy. A two-year follow-up study of the multicenter clinical trial comparing pneumatic retinopexy with scleral buckling. Ophthalmology. 1991;98(7):1115-23.

19. Yokoyama T, Kanbayashi K, Yamaguchi T. Scleral buckling procedure with chandelier illumination for pediatric rhegmatogenous retinal detachment. Clin Ophthalmol. 2015;9:169-73.

20. Skondra D, Westerfeld C, Vavvas DG. Modified controlled encircling scleral buckle for retinal detachment. J Vitreoretin Dis. 2017;1(5):314-6.

21. Park SW, Kwon HJ, Kim HY, Byon IS, Lee JE, Oum BS. Comparison of scleral buckling and vitrectomy using wide angle viewing system for rhegmatogenous retinal detachment in patients older than 35 years. BMC Ophthalmol. 2015;15:121-121.

22. Park SW, Lee JJ, Lee JE. Scleral buckling in the management of rhegmatogenous retinal detachment: patient selection and perspectives. Clin Ophthalmol. 2018;12:1605-15.

23. Kovacevic AR, Stefanovic I, et al. Anatomical and functional factors influencing the results of sclera buckling procedure for macula-off rhegmatogenous 
retinal detachments. Vojnosanit Pregl. 2017;74(3): 212-8.

24. La Heij EC, Derhaag PFJM, Hendrikse F. Results of scleral buckling operations in primary rhegmatogenous retinal detachment. Doc Ophthalmol. 2000;100(1):17-25.

25. Glaser BM, Vidaurri-Leal J, Michels RG, Campochiaro PA. Cryotherapy during surgery for giant retinal tears and intravitreal dispersion of viable retinal pigment epithelial cells. Ophthalmology. 1993;100(4):466-70.

26. Campochiaro PA, Kaden IH, Vidaurri-Leal J, Glaser BM. Cryotherapy enhances intravitreal dispersion of viable retinal pigment epithelial cells. Arch Ophthalmol. 1985;103(3):434-6.

27. Wilkinson CP, Bradford RH Jr. Complications of draining subretinal fluid. Retina. 1984;4(1):1-4.

28. Schwartz SG, Kuhl DP, McPherson AR, Holz ER, Mieler WF. Twenty-year follow-up for scleral buckling. Arch Ophthalmol. 2002;120(3):325-9.

29. Blackorby BL, Jeroudi AM, Blinder KJ, Shah GK. Epiretinal membrane formation after treatment of retinal breaks: cryoretinopexy versus laser retinopexy. Ophthalmol Retina. 2019;3(12):1087-90. https://doi.org/10.1016/j.oret.2019.06.015. 